\title{
Desafios da metodologia de cuidado humanitude na assistência aos idosos institucionalizados em tempos de pandemia
}

Challenges of humanitude care methodology in assist institutionalized elderly people in pandemic times

\section{Desafíos de la metodología del cuidado de la humanitud en la asistencia a las personas mayores en tiempos de pandemia}

ORCID: https://orcid.org/0000-0001-7795-4230

Universidade do Estado de Santa Catarina, Brasil

E-mail: marta.kolhs@udesc.br

Andrea Temponi dos Santos

ORCID: https://orcid.org/0000-0001-7897-8111

Faculdade de Medicina de Jundiaí. Brasil

E-mail: atemponi@gmail.com

Liliana Vanessa Lucio Henriques

ORCID: https://orcid.org/0000-0001-7648-7626 Instituto Gineste-Marescotti, Portugal

E-mail: lilianahenriques312@gmail.com

Maria Amélia Meira

ORCID: https://orcid.org/0000-0002-3532-5548

Universidade Federal de Juíz de Fora, Brasil

E-mail: ameliameira@terra.com.br

Micaela Elizane Bartz Radtke

ORCID: https://orcid.org/0000-0001-6163-3846 Estratégia de Saúde da Família, Prefeitura Municipal de

São Lourenço do Sul (Conveniada), Brasil E-mail: micaelibartz@hotmail.com

Maira Buss Thofehrn

ORCID: https://orcid.org/0000-0002-0864-3284 Universidade Federal de Pelotas, Brasil E-mail: maira.buss@ufpel.edu.br

\footnotetext{
Resumo

Objetivos: conhecer os desafios da $\mathrm{MCH}$ na assistência aos idosos institucionalizados em tempos de pandemia. Metodologia: estudo descritivo, exploratório de abordagem qualitativa com participação de oito profissionais da saúde, de diferentes regiões de Portugal, com formação em MCH. Os dados foram coletados no período de mês de agosto de 2020, através de entrevista semiestruturada individual. Utilizou-se a análise de conteúdo de Bardin (2016). Resultados: os desafios identificados foram o isolamento social e físico, uso de equipamentos de proteção individual (EPI's), falta de profissionais da saúde especializados em $\mathrm{MCH}$, medo de contaminar e de ser contaminado. Conclusão: a pandemia trouxe desafios aos profissionais de saúde que cuidam de idosos institucionalizados, em destaque é evidenciado a insegurança frente ao desconhecimento, no caso da COVID-19. A MCH demonstrou ser uma ferramenta indispensável na assistência aos idosos, ao permitir uma relação intencional e fortalecer os vínculos entre idosos e profissionais de saúde, dentre eles a enfermagem.

Palavras-chave: Metodologia de cuidado humanitude; Epidemia pelo novo Coronavírus (COVID-19); Profissionais de
} saúde; Enfermagem; Assistência a idosos. 


\begin{abstract}
Objectives: to know the challenges of Humanitude Care Methodology in assisting institutionalized elderly in times of pandemic. Methodology: descriptive, exploratory study with a qualitative approach with the participation of eight health professionals from different regions of Portugal, trained in HCM. Data were collected during the month of August 2020, through individual semi-structured interviews. A content analysis by Bardin (2016) was used. Results: the challenges identified were social and physical isolation, use of personal protective equipment (PPE), lack of health professionals specialized in HCM, fear of contaminating and being contaminated. Conclusion: a pandemic brought challenges to health professionals who take care of institutionalized elderly, highlighting the insecurity of ignorance, in the case of COVID-19. The HCM defines itself as an indispensable tool in elderly care, as it allows an international relationship and strengthens the bonds between the elderly and health professionals, including nursing.

Keywords: humanitude care methodology; epidemic by the new Coronavirus (2019-nCoV); Health professionals; nursing; assistance to the elderly.
\end{abstract}

\title{
Resumen
}

Objetivos: conocer los desafíos de la Metodología del Cuidado de la Humanitud en la asistencia a ancianos institucionalizados en tiempos de pandemia. Metodología: estudio descriptivo, exploratorio con enfoque cualitativo con la participación de ocho profesionales de la salud de diferentes regiones de Portugal, formados en SMI. Los datos fueron recolectados durante el mes de agosto de 2020, por medio de entrevistas semiestructuradas individuales. Se utilizó un análisis de contenido de Bardin (2016). Resultados: los desafíos identificados fueron aislamiento social y físico, uso de equipos de protección personal (EPP), falta de profesionales de la salud especializados en $\mathrm{MCH}$, miedo a contaminar y contaminarse. Conclusión: una pandemia trajo desafíos a los profesionales de la salud que atienden a ancianos institucionalizados, destacando la inseguridad del desconocimiento, en el caso del COVID-19. El SMI lo define como una herramienta indispensable en el cuidado de las personas mayores, ya que permite una relación internacional y fortalece los vínculos entre las personas mayores y los profesionales de la salud, incluida la enfermería.

Palabras clave: Metodología del cuidado de la humanidad; Epidemia por el nuevo coronavirus (COVID-19); Profesionales de la salud; Enfermería; Asistencia a los ancianos.

\section{Introdução}

Em 2020 surgiu a doença denominada de COVID- 19, causada por um vírus da família coronavírus ou SARS-CoV-2, que apresenta como sintomatologia febre, tosse e dificuldade respiratória. A transmissão se dá de pessoa a pessoa, através de gotículas, que propagam o vírus para objetos e superfícies, que ficam ativos por horas ou dias. Pelo fato de ser altamente contagiosa, mudanças foram adotadas em todos os países seguindo as orientações da Organização Mundial de Saúde (Yang et al., 2020; Zhang, Wu, Zhao \& Zhand, 2020).

Para prevenir a contaminação foram utilizadas medidas como o confinamento e o distanciamento social, que trouxeram impactos negativos as pessoas idosas, como sentimentos de abandono, devido à ausência das visitas de familiares, da adoção de rígidos protocolos para a assistência em instituições de saúde. O que gera insegurança e medo a todos os envolvidos no cuidado.

Para amenizar os efeitos advindos do COVID-19, os profissionais de saúde precisavam se reinventar e buscar alternativas para diminuir os efeitos negativos na assistência a idosos de modo a minimizar o sofrimento humano. Neste âmbito, a Metodologia de Cuidado Humanitude $(\mathrm{MCH})$, pode ser uma referência na assistência à saúde destas pessoas, que estão em situação de grande vulnerabilidade, por permitir a operacionalização da humanização e dignificação da pessoa (Melo et al. 2019). A MCH é reconhecida em diversos países como França, Suíça, Bélgica, Luxemburgo, Alemanha, Canadá, Portugal, Estados Unidos e Japão, com evidências científicas de melhoria da qualidade de vida dos idosos com doenças crônicas degenerativas, situações de fragilidade e processos de morte e morrer.

Esta metodologia proporciona o cuidado a cada pessoa de forma individual ao respeitar as suas vontades, desejos, além de proporcionar sentido e prazer a todos os atos do viver enquanto a vida existir, ou seja, coloca o foco na interação com a pessoa (Figueiredo, Melo \& Ribeiro, 2018).

A Metodologia de Cuidado Humanitude (MCH), também conhecida como Metodologia de Cuidado GinesteMarescotti® (MGM®), em desenvolvimento desde a década de 70, inclui três pilares relacionais (o olhar, a palavra e o toque) e um pilar identitário, a verticalidade. Tal metodologia operacionaliza-se através de uma sequência estruturada de procedimentos 
cuidativos Humanitude (SEPCH), que se sistematiza em cinco etapas: (1) pré-preliminares, (2) preliminares, (3) rebouclage sensorial, (4) consolidação emocional, e (5) reencontro (Figueiredo, Melo \& Ribeiro, 2018).

Os pré-preliminares consistem no anúncio da presença dos cuidadores ao abrir os canais relacionais, evitar abordagens súbitas, respeitar a privacidade e autonomia do paciente. Os procedimentos preliminares possibilitam o estabelecer da relação através do olhar, da palavra e do toque, pilares relacionais da $\mathrm{MCH}$. A rebouclage sensorial adentra na prestação dos cuidados de modo à consolidação emocional em que se estimula a construção de conhecimentos ao de ficar na memória da pessoa cuidada uma relação positiva estabelecida durante o cuidado, o que facilita a aceitação dos próximos cuidados.

O reencontro é o momento da finalização da relação, e quando se estabelece o compromisso dos futuros cuidados. Nesta etapa abriga-se também, a despedida e marca-se um novo encontro, ameniza a sensação e sentimento de abandono (Henriques, Dourado, Melo \& Tanaka, 2019).

Vale destacar que os princípios da MCH são ferramentas indispensáveis na orientação do fazer profissional os quais desenvolvem uma visão ética, humanista e baseada na evidência (Melo et al., 2019), reduz problemas emocionais e aumenta a satisfação profissional (Henriques et al., 2019). Desta forma o objetivo deste artigo é conhecer os desafios da MCH na assistência aos idosos institucionalizados em tempos de pandemia.

\section{Metodologia}

Estudo descritivo com delineamento qualitativo, desenvolvido com oito profissionais da área de saúde de várias regiões de Portugal, selecionados a partir de critérios de inclusão: formação em MCH e experiência em cuidar de pessoas institucionalizadas durante a pandemia COVID-19. A coleta de dados foi realizada durante o mês de agosto de 2020, através de entrevista semiestruturada individual, com duração aproximada de uma hora.

A saturação teórica do conteúdo em cada categoria ocorreu quando novos elementos deixaram de surgir dos dados. Para saturação teórica, foram seguidos cinco passos: 1- Registro de dados brutos, ao ser realizada a transcrição das respostas obtidas por escrito. 2 - Imersão nos dados, através da leitura flutuante dos dados obtidos. 3 - Compilação das análises individuais e agrupamento por categorias organizadas por meio da codificação colorimétrica. 4- Alocação das categorias, subcategorias e enunciados em uma tabela, para permitir a identificação da regularidade dos achados, de acordo com as categorias e a verificação da consistência dos enunciados. 5- Constatação da saturação teórica dos dados por meio da identificação de ausência de elementos novos em cada categoria (Bardin, 2016). A validade e fidedignidade da análise de conteúdo foi assegurada, por dois codificadores, peritos em pesquisa qualitativa que validaram os achados. Aos participantes foi também dada a oportunidade de darem o seu feedback sobre os achados.

No decurso deste estudo foram respeitadas as considerações ético-legais. Esta investigação obteve o parecer favorável n ${ }^{\circ}$ P688106-2020 da Comissão de Ética da Unidade de Investigação em Ciências da Saúde: Enfermagem da Escola Superior de Enfermagem de Coimbra. Salienta-se o caráter voluntário da participação dos profissionais, tendo sido assinada uma declaração de consentimento informado. Foi garantido o sigilo quanto à identificação dos participantes, sendo os nomes dos entrevistados preservados utilizando-se a letra P (Profissional seguido pelo número sequencial - P1, P2, P3...).

Os dados obtidos foram analisados recorrendo à técnica de análise de conteúdo de natureza temática categorial, seguindo os princípios de homogeneidade, exaustividade, exclusividade, objetividade, e pertinência para a formulação de categorias (Bardin, 2016). O processo de análise de conteúdo iniciou-se com uma análise de todo o material obtido, a qual, seguiu-se a exploração do material com a identificação dos temas, que emergiram das questões. Os dados de cada tema foram organizados em categorias e subcategorias. Foi realizada a codificação com atribuição de códigos específicos a unidades de registo com um determinado teor semântico previamente especificado pelo pesquisador. Estas unidades de registo foram organizadas com a 
codificação dos segmentos das respostas obtidas, cujo conteúdo se mostrou como unidade base e teve o objetivo de fazer a contagem da frequência.

\section{Resultados e Discussão}

Participaram neste estudo oito profissionais, sendo cinco do sexo feminino, três do sexo masculino. As idades variaram entre 30 e 60 anos, sendo com maior frequência dos 40 anos. Todos os participantes tinham como habilitação curso superior nas seguintes áreas: quatro enfermeiros, dois gestores, um médico, uma assistente social. Todos tinham acima de 10 anos de experiência com a $\mathrm{MCH}$.

Da análise emergiram quatro categorias:(a) isolamento social e físico em tempos de pandemia; (b) dificuldades decorrentes da utilização contínua dos EPI's; (c) carência de profissionais com formação na metodologia Cuidado Humanitude; (d) medo. Em cada categoria foram identificadas subcategorias (Tabela 1).

Tabela 1 - Desafios da Metodologia Cuidado Humanitude.

\begin{tabular}{|c|c|c|c|}
\hline Categorias & Subcategorias & $\begin{array}{c}\text { Passagens de } \\
\text { discursos }\end{array}$ & $\begin{array}{c}N^{\circ} \text { de } \\
\text { entrevistas }\end{array}$ \\
\hline \multirow{2}{*}{$\begin{array}{l}\text { Isolamento social e físico em } \\
\text { tempos de pandemia }\end{array}$} & 1. Social & 05 & 05 \\
\hline & 2 Físico & 07 & 03 \\
\hline \multirow[t]{2}{*}{$\begin{array}{l}\text { Dificuldades decorrentes da } \\
\text { utilização contínua de EPI's }\end{array}$} & $\begin{array}{l}\text { 1.Barreira que dificulta a } \\
\text { comunicação. }\end{array}$ & 05 & 05 \\
\hline & 2. Dificuldade no toque & 04 & 03 \\
\hline \multirow[t]{3}{*}{$\begin{array}{l}\text { Carência de profissionais com } \\
\text { formação em MCH }\end{array}$} & $\begin{array}{l}\text { 1.Carência de profissionais com } \\
\text { formação em Humanitude. }\end{array}$ & 02 & 02 \\
\hline & $\begin{array}{l}\text { 2.Formação transversal a todos os } \\
\text { cidadãos }\end{array}$ & 01 & 01 \\
\hline & $\begin{array}{l}\text { 3.Adaptação dos conhecimentos ao } \\
\text { contexto de pandemia }\end{array}$ & 04 & 04 \\
\hline \multirow[t]{2}{*}{ Medo } & 1.Ser contaminado & 05 & 04 \\
\hline & 2.Contaminar & 02 & 01 \\
\hline
\end{tabular}

Fonte: Dados da Pesquisa (2020).

\subsection{Isolamento social e físico em tempos de pandemia}

O isolamento social provocado pela pandemia mostrou-se na abrangência de estar sem convívio interpessoal em todos os relacionamentos humanos.

Por isso, o isolamento social foi identificado como um desafio, conforme os seguintes depoimentos "o distanciamento social [...] sim é um desafio!” (P1; agosto/2020)." .... o isolamento social tem consequências a nível físico e cognitivos, porque a pessoa não percebe a necessidade deste isolamento" (P3, agosto/2020) e "uma série de obstáculos na relação, como o distanciamento social e o isolamento (P5, agosto/2020).

O isolamento físico trouxe sofrimento a todos os envolvidos no cuidado, salientados nos discursos "as pessoas não podem receber o abraço nem do familiar, nem dos amigos... nos cria uma barreira na aproximação” (P4; agosto/2020) “Quando 
aquilo que preconizávamos era a relação social, a proximidade e o estar com o outro, de repente vem a necessidade do isolamento..." (P2; agosto 2020).

Nesse contexto verifica-se que inclusive nas abordagens entre profissional e pacientes, os equipamentos protetores da disseminação do coronavírus que precisam ser utilizados, considerando que todos podem fazer parte do processo de disseminação vírus, foram vistos como impedimento das relações humanas.

\subsection{Dificuldades decorrentes da utilização contínua dos EPI's}

No decorrer da pandemia, protocolos e EPI's diferenciados foram colocados à disposição dos profissionais, como máscara N95, óculos, protetores faciais e luvas, buscando garantir a saúde da força de trabalho na luta contra a COVID-19, nos estabelecimentos de saúde em todo o mundo.

O uso contínuo dos EPI's foram interpretados nos relatos como um entrave nas diversas maneiras do ser humano interagir e foram considerados como uma barreira de comunicação, como enunciam os discursos "Com a pandemia, tudo está mais técnico e mais frio, devido aos EPI's...”(P1; agosto/2020),“Os materiais de proteção individual, escondem as faces, afastando-nos, ... limitando as formas de comunicar, ...o som sai distorcido, com a máscara pode não perceber que estamos a falar com ela...os óculos e a viseira dificultam o olhar no outro, por ficarem embaciados" (P4; agosto/2020) "Tenho uma máscara na frente de minha boca, que dificulta a comunicação...”(P2; agosto/2020) “a paramentação e o uso da máscara... levam as pessoas a limitar as relações e comunicação" (P5; agosto/2020).

As instituições prestadoras de serviços de saúde, em razão da rápida propagação do vírus, fizeram alterações nos fluxos de trabalhos, criaram protocolos de atendimento em saúde e orientações para o uso intensivo dos EPI's. Assim sendo, o equipamento cuja finalidade é impedir a propagação da COVID-19, também dificultou a atitudes de afetividade entre as pessoas. Evidenciaram as dificuldades no toque devido ao uso dos EPI's "os EPI's usados atualmente limitam o toque... (P6; agosto/2020), "torna-se uma barreira física, impedindo o contato...o toque, e, por as vezes, por três pares de luvas" (P4; agosto/2020).

Nesse contexto, apesar de considerarem os EPI's como barreira relacional, os profissionais conferem importância singular e que os utilizavam na sua prática cotidiana. E é esta circunstância que requer vislumbrar novas possibilidades para o exercício do cuidado prestado, afinal, se o toque das mãos está armado de luvas, o olhar deve estar desarmado para possibilitar o encontro de humanidades, e buscar satisfação entre a pessoa cuidada e os profissionais que prestam cuidados.

\subsection{Carência de profissionais com formação na Metodologia Cuidado Humanitude}

Os discursos destacam a carência de profissionais qualificados em $\mathrm{MCH}$ para atuar nas instituições "O que fragiliza a assistência é a falta de profissionais qualificados e com conhecimentos da metodologia de cuidado Humanitude", "Deveria ocorrer no momento formativo do profissional" (P6; agosto/2020). Sabe-se que a pandemia colocou em xeque os sistemas de saúde do mundo, além disso, a formação profissional.

Por outro lado, a MCH é reconhecida pelos participantes como uma estratégia educacional quando relata que "A MCH devia ser ensinada em todo o contexto educacional, aprender a olhar nos olhos, a tocar as pessoas a ouvi-las e respeitar no seu tempo e nas suas capacidades". "As crianças precisam aprender" (P6, agosto/2020). Evidenciam-se nas falas que a MCH é uma metodologia replicável em qualquer contexto de saúde, em todo o ciclo vital e com diferentes patologias. A pandemia demonstrou a necessidade de adaptação dos conhecimentos, tal como segue "Necessidade tornou-se real, é uma luta constante, a instituição Humanitude sentiu o apelo dos cuidadores a pedir mais formação" (P1; agosto/2020), "Houve uma mudança. Tivemos que nos adaptar por algo que não esperávamos. "Portanto, as instituições têm que gerir estes aspectos, desafios que as instituições tiveram que lidar neste momento e adaptar" (P3; agosto/de 2020). 


\subsection{Medo}

Medo pessoal e profissional.

Na categoria medo, emergiram duas subcategorias: ser contaminado e contaminar. Segundos os relatos, constantemente "somos bombardeados com tantas informações que recebemos diariamente, que acabamos por limitar as relações e a comunicação por medo de contaminação" (P5; agosto/2020). "Temos temores de contaminação, se comparar com outras realidades eu sinto que a medicina acaba por ser menos interventiva, restringindo o contacto" (P1; agosto/2020). Além do medo de contaminar-se tem medo de contaminar os idosos sob seus cuidados "É importante esse isolamento de contato, para barrar a infecção porque receio de passar a doença para alguém" (P2; agosto/ 2020); “A pandemia nos transformou, reforçou a importância de conhecer como se equipam e como se desequipam os EPI's, para não colocarmos em risco os que estão a ser cuidados, ..." (P2; agosto/2020).

\section{Discussão}

A pandemia trouxe mudanças significativas a saúde de todos, o que causou perplexidade pelo isolamento físico e social, no qual todos de uma ou de outra forma sentiram-se fragilizados por uma doença desconhecida, a COVID-19. Não diferente do mundo, e com vistas a diminuir os impactos da pandemia, a incidência e a mortalidade, Portugal obedeceu a orientações da Organização Mundial de Saúde, e as autoridades direcionaram a população para realização do isolamento social, físico e o confinamento (SNS, 2020). O distanciamento social visa à diminuição da interação entre as pessoas de uma comunidade para diminuir a velocidade da transmissão do vírus, especialmente quando há pessoas infectadas ainda assintomáticos. Já, o isolamento físico visa separar as pessoas doentes, das pessoas sadias, de modo a evitar a transmissão e a propagação do vírus (Wilder-Smith \& Freedman, 2020).

A tomada de decisão pelo isolamento social, ou seja, de restrição ao convívio social, trouxe prejuízos a saúde mental, especialmente aos idosos, que se tornaram mais vulneráveis e ansiosos. Em concordância com esta afirmação, diferentes estudos apontam que o distanciamento social e o confinamento agravaram o sofrimento das pessoas, não apenas porque a rotina foi modificada, mas porque estão mais submetidos ao estresse, resultantes das restrições de liberdade e dos movimentos o que interfere na capacidade de comunicar-se e desencadeia reações negativas e comportamentais (Zandifar \& Badrfam, 2020).

Para os idosos, o isolamento, a ansiedade, o estresse e a depressão apresentaram gradual aumento nos quadros de demência neste período, o que gera inúmeras preocupações aos profissionais da saúde. Outras repercussões psicológicas negativas do isolamento social manifestam através da raiva, tristeza, preocupação, delírio e confusão que se acentuaram com o isolamento social. Além disso, ocorreram, porém com menor frequência, pesadelos, problemas de higiene, comportamento autodestrutivo, perda cognitiva e queixas psicossomáticas (Silva, Viana \& Lima, 2020). Num olhar mais apurado, envelhecer é uma etapa de inúmeras transformações e limitações, como a perda de papéis sociais e afastamento social, o isolamento intensificou a solidão porque a rede social fomenta vínculos de amizade o que impede o desencadeamento da ansiedade, depressão e sentimentos de abandono. Nesta perspectiva a rede social é de fundamental importância para a saúde dos idosos com implicações direta na sua qualidade de vida, compromete a autonomia e agrava as doenças preexistentes. (Gatto et al., 2018).

O isolamento físico implica na separação das demais, com as quais não mantém contato físico. Manter-se isolado, sem contato físico tem consequências nefastas na qualidade de vida dos seres humanos, o que provoca mudanças no padrão de sono, elevação da pressão arterial, aumento do hormônio cortisol, alterações na função das células e da imunidade corporal, aumento da depressão e dos níveis de bem estar subjetivo global (J. T. Cacioppo, Cacioppo \& Boomsma, 2014). Assim, esses autores revelam que o isolamento físico, ainda promove uma ruptura nas três principais dimensões dos relacionamentos sociais: a conectividade intima a conectividade relacional e a conexão coletiva. Todas são essenciais para o ser humano, pois refere-se 
respectivamente, a sensação de ter alguém na vida, a manutenção de contatos mutuamente gratificantes e ao sentimento de pertencer a um grupo. Neste cenário é importante ressaltar que os reclusos possuem menos genes ativos na proteção contra o vírus (Ayaniam, 2020). Desta forma, a apropriação dos pilares relacionais do olhar, da palavra e do toque pelos profissionais de saúde podem fazer a diferença neste período de distanciamento e confinamento, porque fortalece a humanização no cuidado, o que diminuiu os sentimentos negativos tanto dos idosos como dos profissionais (Melo et al., 2017).

Conceituado como proteção, mas, por vezes entendido como empecilho ao contato humano, os equipamentos de proteção individual (EPI’s) são indicados para proteção de riscos capazes de ameaçar a saúde e a segurança das pessoas e está descrito nos protocolos de prevenção da disseminação do coronavírus, independentes do seu local de trabalho (Ministério da Saúde, 2020). Portanto, os EPI's, protegem a pessoa e o profissional, envolvidos no cuidado, mas, podem dificultar que a pessoa veja claramente, ouça e sinta o toque de quem está cuidando. Com a criação de uma barreira física, como é o caso dos EPI's, os profissionais também podem apresentar mudanças na forma como interagem com os pacientes, tornando-a negativa.

Assim, faz-se necessário uma assistência pautada nos sentimentos de respeito, autonomia, empatia e compaixão, que melhore os resultados da assistência aos idosos o que pode levar a maior satisfação profissional. E ao abolir o cuidado rotineiro, pautar o cuidado com habilidades relacionais, e mediante a interação, com a possibilidade de estimular o idoso na tomada de decisão, torna-se possível envolvê-lo no processo de cuidar, o que permite a participação de todos. (Figueiredo, Melo \& Ribeiro, 2018). Em trabalho com estudantes de enfermagem foi demonstrado que a dificuldade de interação com a pessoa cuidada, se deve ao déficit de profissionais de saúde com formação em humanitude (Melo, Queirós, Tanaka, Henriques \& Neves, 2020). Entretanto, existe uma lacuna importante para a implantação da MC em instituições de cuidado aos idosos, que é justamente a falta de profissionais qualificados na área de geriatria e gerontologia e com formação na metodologia. Os profissionais com formação em $\mathrm{MCH}$ tem a percepção da importância dessa metodologia e de sua aplicabilidade e apresentam maior intencionalidade na relação, facilidade na prestação dos cuidados e na avaliação da eficácia da assistência (Henriques et al., 2019).

No entanto, a pandemia trouxe para o contexto do cuidado uma olhar técnico e uma das grandes dificuldades encontradas em todo o mundo, para o enfrentamento da COVID-19 foi a contratação de profissionais qualificados, aptos em identificar, tomar decisões e a tratar, porque a doença é muito mais grave do que uma insuficiência respiratória, pois, leva a uma liberação de citocinas, principalmente IL-6, com intensa resposta inflamatória, o que ocasiona choque séptico e fenômenos tromboembólicos relacionados à coagulação intravascular disseminada (Rothan \& Byrareddy, 2020).

Entretanto, como seres de relações e gregários, as pessoas precisam do contato, do afeto, porque o outro confirma sua existência no mundo. Neste momento em que o adversário é implacável, formas de comunicação devem ser utilizadas para que os movimentos do corpo, o toque das mãos, o olhar nos olhos, possibilitem a interação e fortaleçam a relação, de modo a tornálas mais frutíferas. A apropriação destes pilares e o contínuo aperfeiçoamento dos procedimentos técnico-relacionais, possibilita atingir a humanização dos cuidados. Trabalhar com saúde, em tempo de pandemia além de sólidos conhecimentos teóricos e práticos, demanda de uma metodologia que possibilite uma assistência que cuide do ser humano doente (Melo et al., 2017).

Cabe ressaltar, que a mensagem precisa ser enviada de forma harmônica e que respeite a identidade, intimidade, autodeterminação e autonomia da pessoa cuidada, além da necessidade de preservar pelo menos dois pilares da MCH, toque e a palavra ou toque e olhar, mas, se possível manter os três pilares no cuidado (Honda, Ishikawa, Takebayashi \& Tierney, 2016). Nesta assertiva, os profissionais devem identificar e avaliar os riscos, bem como as medidas que devem ser tomadas em cada unidade de cuidado, para minimizar os danos durante a estada na instituição, bem como, todos os envolvidos no cuidado estejam cientes das orientações (Teixeira et al, 2020), ao expor a importância da palavra nos relacionamentos humanos. A palavra dentro da $\mathrm{MCH}$ é o pilar que permeia todas os procedimentos de cuidados humanitude.

A rotatividade dos enfermeiros evidência a necessidade de estímulo na formação acadêmica dos profissionais de saúde 
que extrapole os sólidos conhecimentos técnico científicos inerentes à sua profissão, e seja modulada pela formação $\mathrm{MCH}$ o que pode transformar a assistência ao prescrever e supervisionar as práticas cuidativas (Melo et al., 2019). Desta forma, os profissionais qualificados em $\mathrm{MCH}$ seriam de fato agentes de mudanças na aplicação das técnicas relacionais e poderiam colocar na prática de cuidados a verticalização, o autocuidado e a promoção da autonomia, para todas as pessoas em qualquer espaço de cuidado a saúde.

Entretanto, a MCH ainda enfrenta resistência, por expor mudanças cruciais em conhecimento tradicionais, que foram passados de geração em geração, o que exige também vontade de mudanças de todos os envolvidos, inclusive dos gestores (Figueiredo, Melo \& Ribeiro, 2018). A MCH é instrumento metodológico inovador comprovado em âmbito nacional e internacional que capacita os profissionais com técnicas relacionais com vistas a reduzir as dificuldades na prestação dos cuidados e que traz impactos positivos no bem-estar dos envolvido (Melo et al., 2017).

As ações de cuidado durante a pandemia da COVID-19 trouxeram outras inquietações como o medo. O medo é um mecanismo de defesa fundamental para a sobrevivência e potencializa vários processos biológicos em resposta a uma ameaça ou a eventos ameaçadores. De outro modo, ele intensifica as reações de ansiedade e de estresse em pessoas saudáveis e agrava os sintomas em quem sofre de algum transtorno psiquiátrico pré-existente, especialmente no atual momento da pandemia. Neste contexto, os profissionais de saúde envolvidos na assistência direta aos pacientes infectados são expostos diariamente a elevadas cargas virais, além de enorme estresse (Silva, Viana \& Lima, 2020).

O medo da contaminação pelo coronavírus tem gerado afastamento do trabalho, doença e morte, além de intenso sofrimento psíquico. É possível identificar a forte associação entre aumento da jornada de trabalho, inadequada higienização das mãos e o risco de infecção (Yang et al., 2020). Ainda, profissionais quando exaustos e sobrecarregados após longas exaustivas horas de trabalho descuidam-se e se expõem ao risco de contaminação (Huang, Lin, Tang, Yu \& Zhou, 2020). A escassez de equipamentos EPI's também intensifica e exacerba o medo da exposição à contaminação pelo coronavírus, bem como, o sofrimento psicológico dos profissionais da saúde (Ayanian, 2020).

Ser um profissional de saúde, exigem sólidos conhecimentos técnico científicos que permitam cuidar da saúde humana em diferentes situações. Entretanto, a pandemia escancarou a fragilidade do tecido social e respostas diferenciadas podem vir dos profissionais de saúde emanadas do medo de morrer, da perda de pessoas próximas, das perdas dos meios de subsistência e da exclusão social associada a doenças. As pressões e preocupações são causadoras de estresse emocional, no qual o medo e a angústia podem influenciar na diminuição da imunidade determinando mudanças na manutenção da saúde física e mental (Ayanian, 2020; Yang et al, 2020; Huang, Lin, Tang, Yu \& Zhou, 2020).

Alguns estudos identificaram níveis elevados de angústia nos enfermeiros, devido à sensação de perda do controle da situação, receito pela sua saúde e de propagar a doença, além de sinais e sintomas psicológicos que incluíam estresse, ansiedade e depressão (Huang, Lin, Tang, Yu \& Zhou, 2020; Yang et al., 2020). Ainda, os profissionais que trabalham diretamente com os pacientes infectados com COVID-19, manifestam preocupações em infectar os membros da família, especialmente os mais velhos, os imunocomprometidos e aqueles com doenças graves (Ayanian, 2020). Os profissionais da linha de frente na assistência aos acometidos da COVID-19, precisam também estar atualizados nos diferentes aparelhos tecnológicos de ponta, pois vivenciam situações limites, convivendo com o medo de adoecer e morrer e de transmitir a doença a seus familiares (Barbosa, M.P. Gomes, Souza, \& Gomes, 2020). Nesse sentido os profissionais precisam ser cuidados por uma equipe multidisciplinar.

\section{Conclusão}

A pandemia evidenciou a fragilidade dos profissionais de saúde dos idosos frente a uma doença desconhecida, nesse novo contexto de cuidado. Destarte, esse estudo permitiu conhecer os desafios da $\mathrm{MCH}$ na assistência aos idosos 
institucionalizados em tempos de pandemia. Dentre eles o isolamento físico trouxe sofrimentos a todos os envolvidos no cuidado e a decisão do isolamento social intensificou os agravos à saúde mental, especialmente aos idosos, que se tornaram mais vulneráveis e ansiosos.

As relações também sofreram prejuízo pela utilização dos equipamentos de proteção individual (EPI's), que dificultou a comunicação e o toque. Entretanto, reforçam a necessidade do uso dos EPI's para cuidarem de si próprios devido à morbimortalidade da coronavírus.

Destacou-se ainda como desafio nestes tempos de pandemia, a carência de profissionais qualificados em MCH para atuar nas instituições. Os profissionais também sentiram a necessidade de buscar de novos conhecimentos como auxílio na adaptação e salientam que a qualificação $\mathrm{MCH}$ deveria ser incorporada já na formação do profissional de saúde. O medo de ser contaminado e contaminar também foi observado, e todos de alguma forma manifestaram ansiedade e incerteza frente a pandemia.

O estudo tem limitações devido ao tamanho da amostra ser reduzida, o impossibilita a generalização dos dados obtidos. E devido à resistência que a MCH ainda enfrenta, apesar de ser um instrumento metodológico inovador comprovado em âmbito nacional e internacional, por expor mudanças cruciais em conhecimentos tradicionais, o que exige também vontade de mudanças de todos os envolvidos, inclusive de todos os gestores, aventa-se a necessidade de conhecer o entendimento sobre a MCH com esses líderes institucionais. Para, além disso, sugere-se a realizações de estudos que possam trazer novas evidências da metodologia em diferentes cenários da praticas, que permitam comparar resultados e eficácia do cuidado humanitude em todo o ciclo vital. Porém, este foi um dos momentos para testar os pilares da humanitude. Na prática diária o pilar da palavra tornou-se uma bússola que norteia a relação. A Metodologia de Cuidado Humanitude $(\mathrm{MCH})$ é uma ferramenta indispensável na assistência aos idosos em tempos de pandemia. Salienta-se a importância da manutenção dos pilares relacionais toque, olhar e palavra como alicerce das ações dos profissionais de saúde, e podem fazer a diferença neste período de distanciamento e confinamento, na medida que fortalece a humanização no cuidado, o que pode diminuir os sentimentos negativos dos idosos e dos profissionais.

\section{Referências}

Ayanian, J. Z. (2020). Mental health needs of health care workers providing frontline COVID-19 care: Editor's Comment COVID-1. Jama [Internet]. 2020. Recuperado de https://jamanetwork.com/channels/health-forum/fullarti.

Barbosa, D. J., Gomes, M. P., Souza, F. B. A. de., \& Gomes, A. M. T. (2020). Fatores de estresse nos profissionais de enfermagem no combate à pandemia da COVID-19: síntese de evidências. Comunicação em Ciências da Saúde, 31(Suppl 1), 31-47. Recuperado de http://www.escs.edu.br/revistaccs/index.php/comunicacaoemcienciasdasaude/article/view/651.

Bardin, L. (2016). Análise de conteúdo. São Paulo: Edições 70.

Cacioppo, J. T., Cacioppo, S., \& Boomsma, D. I. (2014). Evolutionary mechanisms for loneliness. Cognition \& emotion, 28(1), 3-21. https://doi.org/10.1080/02699931.2013.837379.

Figueiredo, A., Melo, R., \& Ribeiro, O. (2018). Metodologia de Cuidado Humanitude: dificuldades e benefícios da sua implementação na prática. Revista de Enfermagem Referência. IV (17):1-10. doi: https://doi.org/10.12707/RIV17063.

Gatto, J. M., Zenevicz, L. T., Madureira, V. S. F., Silva, T. G., Souza, S. S., Celich, K. L. S., \& Leo, M. M. F. (2018). Saúde mental e qualidade de vida de pessoas idosas. Revista Enferm. 36(3), 302-10. doi: https://doi.org/10.15446/av.enferm.v36n3.68498.

Henriques, L. V. L., Dourado, M. de A. R.F., Melo, R. C. C.P. de., \& Tanaka, L. H. (2019). Implementation of the Humanitude Care Methodology: contribution to the quality of health care. Rev. Latino-Am. Enfermagem. 27,e3123. Recuperado de http://www.scielo.br/pdf/rlae/v27/pt_0104-1169-rlae-27-e3123.pdf.

Honda, M., Ito, M., Ishikawa, S., Takebayashi, Y., \& Tierney, L. (2016) Reduction of behavioral psychological symptoms of dementia by multimodal comprehensive care for vulnerable geriatric patients in an acute care hospital: A case series. Case Rep Med. 4813196. doi: http:// dx.doi.org/10.1155/2016/481319.

Huang, L., Lin, G., Tang, L., Yu, L., \& Zhou, Z. (2020). Special attention to nurses protection during the COVID-19 epidemic. Crit Care, 24(1), 120. doi: $10.1186 / \mathrm{s} 13054-020-2841-7$.

Jacquard, A. (1986). L’Héritage de La Liberté: De L’animalité à L’Humanitude. Paris: Éditions Seuil. 
Research, Society and Development, v. 10, n. 13, e479101321284, 2021

(CC BY 4.0) | ISSN 2525-3409 | DOI: http://dx.doi.org/10.33448/rsd-v10i13.21284

Melo, R. C. C. P. de., Costa, P. J., Henriques, L. V. L., Tanaka, L. H., Queirós, P. J. P., \& Araújo, J. P. (2019). Humanitude in the humanization of elderly care: experience reports in a health service. Revista Brasileira de Enfermagem, 72(3),825-29. Epub June 07, 2019. doi: https://doi.org/10.1590/0034-7167-2017-0363.

Melo, R. C. C. P. de., Queirós, P. J. P., Tanaka, L. H., Henriques, L. V. L., \& Neves, H. L. (2020) Nursing Students' Relational Skills with Elders Improve through Humanitude Care Methodology. Int. J. Environ. Res. Public Health, 17(22), 8588. https://doi.org/10.3390/ijerph17228588

Melo, R. C. C. P. de, Queirós, P. J. P., Tanaka, L. H., Salgueiro, N. R., Alves, R. E., \& Araújo, J. P. (2017). State-of-the-art in the implementation of journal humanitude care methodology in Portugal. Rev Enf Ref [Internet], 4(13),53-62. doi: https://doi.org/10.12707/RIV17019.

Ministério da Saúde. Protocolo de Manejo Clínico para o Novo Coronavírus (2019-nCoV). Brasília - DF. $1^{\text {a }}$ edição - 2020 - publicação eletrônica

Rothan, H. A. E., Byrareddy, S. N. (2020). A Epidemiologia e Patogênese da Doença coronavírus (COVID-19) Surto. Jornal da Autoimunidade, 109, 102433. doi: https://doi.org/10.1016/j.jaut.2020.102433.

Serviço Nacional de Saúde. Direção Geral de Saúde. COVID 19 (2020). Manual para famílias Saúde República Portuguesa como lidar com o isolamento em contexto familiar, 2020. https://www.dgs.pt/documentos-e-publicacoes/manual-para-familias-como-lidar-com-o-isolamento-em-contexto-familiar-pdf.aspx.

Silva, M. de L., Viana, S. A. A., Lima, P. (2020). Impacto na saúde mental do idoso durante o período de isolamento social em virtude da disseminação da doença covid19: uma revisão literária. Revista Diálogos em Saúde, 3(1) jon/jun. https://periodicos.iesp.edu.br/index.php/dialogosemsaude/article/view/272/232

Teixeira, C. F. de S., Soares, C. M., Souza, E. A., Lisboa, E. S., Pinto, I. C. de M., Andrade, L. R. de., \& Espiridião, M. A. (2020). A saúde dos profissionais de saúde no enfrentamento da pandemia de Covid-19. Ciência \& Saúde Coletiva, 25(9), 3465-3474. Epub August 28, 2020. doi: https://doi.org/10.1590/141381232020259.19562020

Wilder-Smith, A., Freedman, D. O. (2020). Isolation, quarantine, social distancing and community containment: pivotal role for old-style public health measures in the novel coronavirus (2019-nCoV) outbreak. J Travel Med., 27(2),1-4. Doi 10.1093/jtm/taaa020.

Yang, Y., Li, W., Zhang, Q., Zhang, L., Cheung, T., Xiang, Y. (2020). Mental health services for older adults in China during the COVID-19 outbreak. The Lancet Psychiatry, 7(4),e19. doi: https://doi.org/10.1016/S2215-0366(20)30079-1.

Zandifar, A., \& Badrfam, R. (2020). Iranian mental health during the COVID-19 epidemic. Asian Journal of Psychiatry, 51,101990. doi: http://dx.doi.org/10.1016/j.ajp.2020.101990.

Zhang, J., Wu, W., Zhao, X., \& Zhang, W. (2020b). Recommended psychological crisis intervention response to the 2019 novel coronavirus pneumonia outbreak in China: a model of West China Hospital. Precision Clinical Medicine, 3(1),3-8. doi: http://dx.doi.org/10.1093/pcmedi/pbaa006. 